\title{
Farnesol inhibits in vitro growth of the Cryptococcus neoformans species complex with no significant changes in virulence-related exoenzymes
}

\author{
Rossana de Aguiar Cordeiro ${ }^{\text {, }}$ George Cândido Nogueira ${ }^{\text {, }}$, \\ Raimunda Sâmia Nogueira Brilhante ${ }^{a, *}$, Carlos Eduardo Cordeiro Teixeira ${ }^{a}$, \\ Charles Ielpo Mourão ${ }^{a}$, Débora de Souza Collares Maia Castelo-Branco ${ }^{a}$, \\ Manoel de Araújo Neto Paiva ${ }^{a, b}$, Joyce Fonteles Ribeiro ${ }^{a}$, André Jalles Monteiro ${ }^{c}$, \\ José Júlio Costa Sidrim ${ }^{\text {a }}$, Marcos Fábio Gadelha Rocha ${ }^{\text {a,b }}$ \\ ${ }^{a}$ Department of Pathology and Legal Medicine, School of Medicine, Specialized Medical Mycology Center, Postgraduate Program in Medical Microbiology, \\ Federal University of Ceará, Fortaleza, CE, Brazil \\ ${ }^{\mathrm{b}}$ School of Veterinary, Postgraduate Program in Veterinary Science, State University of Ceará, Fortaleza, CE, Brazil \\ ${ }^{\mathrm{c}}$ Department of Statistics and Applied Mathematics, Federal University of Ceará, Fortaleza, CE, Brazil
}

\section{A R T I C L E I N F O}

Article history:

Received 6 November 2011

Received in revised form 4 April 2012

Accepted 5 April 2012

\section{Keywords:}

Farnesol

Cryptococcus neoformans species complex

Minimum inhibitory concentration

Phospholipases

Proteases

\begin{abstract}
A B S T R A C T
Farnesol is a sesquiterpene alcohol that modulates cell-to-cell communication in Candida albicans. In recent years, several studies have shown that this molecule presents inhibitory effects against non-albicans Candida species, Paracoccidioides brasiliensis and bacteria. The present study aimed at determining the effect of farnesol on the growth of strains of the Cryptococcus neoformans species complex, through microdilution assays. In addition, the effect of farnesol on the synthesis of phospholipase and protease - important virulenceassociated enzymes - by C. neoformans and Cryptococcus gattii was also investigated. A total of 36 strains were studied, out of which 20 were from veterinary sources, 8 were from human cases and 8 were from a reference collection. The minimum inhibitory concentrations (MICs) were determined in accordance with the M27-A3 protocol as described by the CLSI and farnesol was tested at a concentration range of $0.29-150 \mu \mathrm{M}$. Phospholipase and protease activities were evaluated through growth on egg yolk agar and spectrophotometry, respectively, after pre-incubating the strains at different farnesol concentrations (MIC/4, MIC/2 and MIC). It was observed that farnesol presents an inhibitory activity against $C$. neoformans and $C$. gattii (MIC range: $0.29-75.0 \mu \mathrm{M}$ ). Although farnesol did not significantly alter phospholipase activity, a tendency to decrease this activity was observed. Concerning protease, no statistically significant differences were observed when comparing the production before and after pre-incubation at different farnesol concentrations. Based on these findings, it can be concluded that farnesol has in vitro inhibitory activity against $C$. neoformans and $C$. gattii, but has little impact on the production of the analyzed virulence factors.
\end{abstract}

(c) 2012 Elsevier B.V. All rights reserved.

\footnotetext{
* Corresponding author at: Rua Barão de Canindé, 210, Montese, CEP: 60.425-540, Fortaleza, CE, Brazil. Fax: +55 8532951736.

E-mail address: brilhante@ufc.br (R.S.N. Brilhante).
}

\section{Introduction}

The Cryptococcus neoformans species complex is formed by two distinct microorganisms, C. neoformans and C. gattii, which are sub-classified into at least two varieties, five serotypes and eight molecular types (Kwon-Chung and Varma, 2006; Bovers et al., 2008; Lester et al., 2011). Despite sharing several phenotypical and molecular 
characteristics, these species differ from each other concerning ecological and epidemiological aspects (Lin and Heitman, 2006; Costa et al., 2010). They are the major causative agents of cryptococcal meningoencephalitis, the most frequent clinical manifestation of cryptococcosis, which has a high mortality rate in developing countries (Kronstad et al., 2011).

To succeed in the host environment, Cryptococcus spp. produce a large amount of virulence factors, which directly influence the infectivity of an individual strain (Ma and May, 2009). Among these main virulence factors, hydrolytic enzymes able to degrade lipids and proteins have gained attention in recent years (Bien et al., 2009; Chayakulkeeree et al., 2011). Phospholipases are directly related to the destabilization of host cell membranes (Ma and May, 2009) and proteases have been shown to degrade several host molecules (Chen et al., 1996).

Even though new antifungal drugs have been developed in recent years, the availability of antifungal agents with anticryptococcal activity is still limited. This scenario has motivated the pursuit of new compounds that present antifungal properties against Cryptococcus spp. Based on this perspective, farnesol, a sesquiterpene alcohol, has been investigated for its antimicrobial potential (JabraRizk et al., 2006; Semighini et al., 2008; Derengowski et al., 2009; Weber et al., 2010; Pammi et al., 2011).

Farnesol is found in plant extracts and also produced by Candida albicans as a quorum sensing molecule (Hornby et al., 2001, Derengowski et al., 2009). This autoregulatory compound alters $C$. albicans morphology and also has a dramatic impact on fungal growth and survival (Shirtliff et al., 2009; Weber et al., 2010). Several studies have shown that this molecule also has inhibitory effects against other pathogens, including non-albicans Candida species (Weber et al., 2010), Paracoccidioides brasiliensis (Derengowski et al., 2009), Fusarium graminearum (Semighini et al., 2008) and bacteria (Pammi et al., 2011; Brilhante et al., 2012).

The present study aimed at evaluating the antifungal potential of farnesol against $C$. neoformans and $C$. gattii. In addition, the effect of farnesol on phospholipase and protease activities was also investigated.

\section{Material and methods}

\subsection{Fungal strains}

A total of 36 strains from the culture collection of the Specialized Medical Mycology Center of Federal University of Ceará were included in this study, out of which 20 were from veterinary sources ( 2 from animal clinical cases and 18 from pigeon excreta) and 8 from human clinical cases. Eight international reference strains of Cryptococcus sp. obtained from the Evandro Chagas Clinical Research Institute, Brazil (IPEC/FIOCRUZ) were used as controls: $C$. neoformans var. grubii WM 148 (serotype A, VNI/AFLP1); $C$. neoformans var. grubii WM 626 (serotype A, VNII/AFLP1A); C. neoformans WM 628 (serotype AD, VNIII/AFLP2); C. neoformans var. neoformans WM 629 (serotype D, VNIV/ AFLP3); C. gattii WM 179 (serotype B, VGI/AFLP4); C. gattii WM 178 (serotype B, VGII/AFLP6); C. gattii WM 175 (serotype B, VGIII/AFLP5); and C. gattii WM 779 (serotype
C, VGIV/AFLP7), as described by Meyer et al. (2003). In addition, Candida parapsilosis ATCC 22019 was included as internal quality control for the susceptibility tests (CLSI, 2008 ). All isolates were stored on $2 \%$ potato dextrose agar supplemented with glycerol, at $-20^{\circ} \mathrm{C}$, and were recovered through growth on $2 \%$ potato dextrose agar at $35^{\circ} \mathrm{C}$, for 2 days (Cordeiro et al., 2011).

\subsection{Dilution of farnesol, amphotericin B and fluconazole}

The strains were tested against farnesol diluted with $30 \%$ dimethyl sulfoxide (DMSO) at the moment of use, in order to obtain a stock solution at a concentration of $1892 \mu \mathrm{M}$. Afterwards, the stock solution was diluted with RPMI 1640 to a concentration of $600 \mu \mathrm{M}$, in which DMSO was at a concentration of approximately $10 \%$. The tested concentration range was from 0.29 to $150 \mu \mathrm{M}$.

Amphotericin B (Sigma Chemical Corporation, Germany) and fluconazole (Pfizer Pharmaceuticals, USA) were tested against $C$. parapsilosis ATCC 22019 as internal quality control, and also against the eight international reference Cryptococcus strains. These drugs were diluted with sterile distilled water, as described by the document M27-A3 of the Clinical Laboratory Standards Institute (CLSI, 2008). The tested concentration ranged from 0.03125 to $16 \mu \mathrm{g} / \mathrm{mL}$ and from 0.125 to $64 \mu \mathrm{g} / \mathrm{mL}$, for amphotericin B and fluconazole, respectively. Additionally, the effect of DMSO on the viability of Cryptococcus spp. was evaluated, at concentrations that ranged from 0.05 to $30 \%$.

\subsection{Testing in vitro susceptibility to farnesol}

Fungal inocula were prepared from cultures grown on potato dextrose agar at $28{ }^{\circ} \mathrm{C}$, for $48 \mathrm{~h}$. Sterile $0.9 \%$ saline $(5 \mathrm{~mL})$ was added to sterile glass slants and a sample of the colony was added to the saline solution, adjusting its turbidity to 0.5 on the McFarland scale (CLSI, 2008). Afterwards, inocula were diluted to $1: 100$ and $1: 20$ with RPMI 1640 medium supplemented with L-glutamine (HiMedia, Mumbai, India), and buffered to $\mathrm{pH} 7$ with $0.165 \mathrm{M}$ morpholinepropanesulfonic acid (MOPS). The final inoculum concentration was $0.5-2.5 \times 10^{3}$ cells $/ \mathrm{mL}$ (CLSI, 2008; Costa et al., 2010).

The minimum inhibitory concentrations (MICs) of farnesol and the tested drugs against $C$. neformans and $C$. gattii strains were determined through the broth microdilution method, in 96-well microdilution trays, with a capacity of $200 \mu \mathrm{L} /$ well. These trays were properly prepared and incubated at $35^{\circ} \mathrm{C}$ for $72 \mathrm{~h}$, when they were visually read. The MIC for farnesol was defined as the lowest drug concentration able to inhibit $80 \%$ of fungal growth, when compared to the farnesol-free control wells (Jabra-Rizk et al., 2006). Concerning amphotericin B and fluconazole, the MICs were defined as the lowest concentrations capable of inhibiting $100 \%$ and $50 \%$ of fungal growth, respectively (CLSI, 2008).

\subsection{Effects on phospholipase and protease activities after exposure to farnesol}

In this step, all strains were grown on $2 \%$ Sabouraud dextrose agar and incubated at $37^{\circ} \mathrm{C}$, for $48 \mathrm{~h}$. Afterwards, 
the strains were cultured in RPMI 1640 medium, containing three decreasing concentrations of farnesol (MIC, MIC/ 2 and $\mathrm{MIC} / 4$ ), at $37^{\circ} \mathrm{C}$, for $48 \mathrm{~h}$. A farnesol-free control for each strain was also included.

Phospholipase activity was evaluated according to Sidrim et al. (2010). Initially, the fungal suspensions in RPMI medium containing different farnesol concentrations were centrifuged at $805 \times \mathrm{g}$. Then, from the fungal pellets, yeast inocula were prepared in $0.9 \%$ saline, reaching turbidity equivalent to 4.0 on the McFarland scale. The medium used was $2 \%$ Sabouraud dextrose agar, supplemented with $1 \mathrm{~mol} / \mathrm{L}$ of sodium chloride, $0.05 \mathrm{~mol} / \mathrm{L}$ of calcium chloride and $8 \%$ sterile egg yolk emulsion at $30 \%$. The emulsion was heated to $40^{\circ} \mathrm{C}$ and incorporated into the sterile Sabouraud medium after it reached a temperature of $40{ }^{\circ} \mathrm{C}$. A volume of $5 \mu \mathrm{L}$ of each inoculum was applied onto a 5-mm sterilized filter paper disk, which was placed on the agar surface. The plates were then incubated at $35^{\circ} \mathrm{C}$ for seven days (Sidrim et al., 2010).

The phospholipase activity $(\mathrm{Pz})$ was determined by calculating the ratio between the diameter of the fungal colony and the total diameter, including the colony and the precipitation zone. When $\mathrm{Pz}=1$, the isolate is phospholipase negative; when $1>\mathrm{Pz} \geq 0.64$ the isolate is positive for phospholipase activity; and when $\mathrm{Pz}<0.64$, the isolate is strongly positive for the enzymatic activity (Price et al., 1982; Sidrim et al., 2010).

For protease production, the analyses were performed according to Cenci et al. (2008), with modifications (Brilhante et al., 2011). Briefly, the strains were incubated in RPMI containing different decreasing farnesol concentrations, under slow agitation of $150 \times \mathrm{g}$, for $48 \mathrm{~h}$, at $28^{\circ} \mathrm{C}$. Afterwards, the material was centrifuged for $15 \mathrm{~min}$ at $805 \times g$ to separate the enzymatic extract from the cells. Then, $2 \mathrm{~mL}$ of the enzymatic extract was incubated at $37{ }^{\circ} \mathrm{C}$ with $2 \mathrm{~mL}$ of $0.3 \%$ azoalbumine for $48 \mathrm{~h}$. After this procedure, the reaction was stopped by the addition of $10 \%$ trichloroacetic acid. To the reaction mixture, $2 \mathrm{~mL}$ of $0.5 \mathrm{M} \mathrm{KOH}$ was added for posterior spectrophotometric analysis at $530 \mathrm{~nm}$. The enzymatic unit (EU) was defined as the amount of enzyme that causes an increase in absorbance of 0.001 per $\mathrm{mL}$ at $530 \mathrm{~nm}$.

\subsection{Statistical analysis}

To analyze the effects of farnesol on the growth of the strains and the activity of virulence factors, the data were analyzed through univariate analysis of variance. Farnesol MICs were evaluated considering species and source of each strain, whereas phospholipase and protease values were analyzed before and after exposure to farnesol. In this analysis, species and source of strains were also considered. $P$-values lower than 0.05 indicated statistically significant differences.

\section{Results}

Farnesol presented in vitro inhibitory effects against the tested strains, with MICs varying from 0.29 to $75 \mu \mathrm{M}$ for both C. neoformans and C. gattii. MIC50 and MIC90 of farnesol against $C$. neoformans were $9.37 \mu \mathrm{M}$ and $75 \mu \mathrm{M}$, respectively; while for $C$. gattii these values were $4.68 \mu \mathrm{M}$ and $75 \mu \mathrm{M}$, respectively. Regarding antifungals, the MICs against $C$. parapsilosis ATCC 22019 were $0.5 \mu \mathrm{g} / \mathrm{mL}$ for AMB and $2 \mu \mathrm{g} / \mathrm{mL}$ for FLC. As for the reference strains of Cryptococcus sp. the MICs ranged from 0.0625 to $1.0 \mu \mathrm{g} / \mathrm{mL}$ for AMB and from 4.0 to $16.0 \mu \mathrm{g} / \mathrm{mL}$ for FLC. Information regarding susceptibility to farnesol of each tested strain and antifungal susceptibility of control strains is shown in Table 1 . Susceptibility tests were performed with DMSO as solvent at $1.25 \%$, which presented no inhibitory effect against Cryptococcus strains.

Furthermore, 18 (50\%) out of the 36 tested strains secreted phospholipases without being exposed to farnesol (control). When the 36 strains were pre-incubated at three different concentrations of this compound, 8 (22.22\%), 11 (30.55\%) and 11 (30.55\%) strains did not exhibit any alteration in phospholipase secretion after exposure to farnesol concentrations of $\mathrm{MIC} / 4, \mathrm{MIC} / 2$ and MIC, respectively (Table 2 ). Under experimental conditions, a reduction in enzymatic activity was observed in 17 (47.22\%), 14 (38.88\%) and 15 (41.66\%) strains after preincubation at MIC/4, MIC/2 and MIC of farnesol, respectively. Increased phospholipase activity was observed in 11 (30.55\%), 11 (30.55\%) and 10 (27.77\%) strains after preincubation at MIC/4, MIC/2 and MIC of farnesol, respectively.

Concerning protease activity, 18 strains (50\%) secreted these enzymes without being exposed to farnesol (control). After pre-incubation at different concentrations of this sesquiterpene, no statistically significant differences were observed between the control and the tested farnesol concentrations. The mean \pm SD values of protease activity (EU) of C. neoformans were as follows: $0.008 \pm 0.013$ for control; $0.017 \pm 0.033$ for $\mathrm{MIC} / 4 ; 0.016 \pm 0.031$ for $\mathrm{MIC} / 2$ and $0.026 \pm 0.033$ for MIC. As for $C$. gattii, the mean values were $0.007 \pm 0.009$ for control; $0.015 \pm 0.024$ for $\mathrm{MIC} / 4$; $0.008 \pm 0.008$ for $\mathrm{MIC} / 2$ and $0.004 \pm 0.008$ for MIC.

\section{Discussion}

The mechanisms through which farnesol acts on fungal cells is still unknown (Derengowski et al., 2009; Langford et al., 2010), but it is believed that it damages the fungal cell membrane and impairs ergosterol synthesis, considering that farnesol and ergosterol share many precursors in the sterol biosynthetic pathway (Hornby and Nickeerson, 2004; Navarathna et al., 2005; Jabra-Rizk et al., 2006). In addition, as an efflux pump modulator, farnesol may affect membrane permeability through the disruption of cytoplasmic membranes (Jin et al., 2010; Sharma and Prasad, 2011).

The present article showed that farnesol can also inhibit growth of $C$. neoformans and $C$. gattii obtained from veterinary sources. The MIC values for both species were lower than those observed for other fungal pathogens, such as $P$. brasiliensis, for which inhibition was achieved at $25 \mu \mathrm{M}$ (Derengowski et al., 2009), and C. parapsilosis, which was inhibited by $50 \mu \mathrm{M}$ of farnesol (Rossignol et al., 2007).

Even though $C$. gattii has been described as being less susceptible to classical antifungal drugs (Trilles et al., 
Table 1

Minimum inhibitory concentration (MIC) of farnesol against strains of the Cryptococcus neoformans species complex.

\begin{tabular}{|c|c|c|c|c|c|c|}
\hline Collection number & Specie & Serotype & Source & FNS & FLC & AMB \\
\hline CEMM 03-2-067 & C. neoformans & A & Veterinary & 0.29 & - & - \\
\hline CEMM-05-1-044 & C. neoformans & A & Human & 0.58 & - & - \\
\hline CEMM-05-1-045 & C. neoformans & A & Human & 0.58 & - & - \\
\hline CEMM-05-1-048 & C. neoformans & A & Human & 2.34 & - & - \\
\hline CEMM-05-3-002 & C. neoformans & A & Veterinary & 2.34 & - & - \\
\hline CEMM 05-1-090 & C. neoformans & A & Veterinary & 2.34 & - & - \\
\hline CEMM-05-1-043 & C. neoformans & A & Human & 4.68 & - & - \\
\hline CEMM-05-1-050 & C. neoformans & A & Veterinary & 4.68 & - & - \\
\hline CEMM-05-1-047 & C. neoformans & A & Human & 9.37 & - & - \\
\hline CEMM-05-1-046 & C. neoformans & A & Human & 18.75 & - & - \\
\hline CEMM-05-1-042 & C. neoformans & A & Human & 18.75 & - & - \\
\hline CEMM-05-3-003 & C. neoformans & A & Veterinary & 37.5 & - & - \\
\hline CEMM 03-2-075 & C. neoformans & A & Veterinary & 37.5 & - & - \\
\hline CEMM 03-2-078 & C. neoformans & A & Veterinary & 37.5 & - & - \\
\hline CEMM-05-1-049 & C. neoformans & A & Human & 75.0 & - & - \\
\hline CEMM-05-3-001 & C. neoformans & A & Veterinary & 75.0 & - & - \\
\hline CEMM 03-2-061 & C. neoformans & A & Veterinary & 75.0 & - & - \\
\hline CEMM 05-3-033 & C. gattii & $\mathrm{B}$ & Veterinary & 0.29 & - & - \\
\hline CEMM 05-2-093 & C. gattii & B & Veterinary & 0.58 & - & - \\
\hline CEMM 05-3-032 & C. gattii & B & Veterinary & 1.17 & - & - \\
\hline CEMM 05-1-099 & C. gattii & B & Veterinary & 1.17 & - & - \\
\hline CEMM-03-2-074 & C. gattii & B & Veterinary & 4.68 & - & - \\
\hline CEMM 03-2-066 & C. gattii & B & Veterinary & 4.68 & - & - \\
\hline CEMM 05-2-087 & C. gattii & B & Veterinary & 4.68 & - & - \\
\hline CEMM 05-3-030 & C. gattii & $\mathrm{B}$ & Veterinary & 18.75 & - & - \\
\hline CEMM 05-2-091 & C. gattii & B & Veterinary & 37.5 & - & - \\
\hline CEMM 03-2-079 & C. gattii & B & Veterinary & 75.0 & - & - \\
\hline CEMM 05-3-031 & C. gattii & B & Veterinary & 75.0 & - & - \\
\hline WM626 & C. neoformans & A & Reference & 0.58 & 4.0 & 1.0 \\
\hline WM148 & C. neoformans & A & Reference & 1.17 & 16.0 & 0.0625 \\
\hline WM629 & C. neoformans & $\mathrm{D}$ & Reference & 2.34 & 8.0 & 0.5 \\
\hline WM628 & C. neoformans & $\mathrm{AD}$ & Reference & 18.75 & 4.0 & 0.5 \\
\hline WM178 & C. gattii & B & Reference & 0.58 & 4.0 & 1.0 \\
\hline WM779 & C. gattii & $\mathrm{C}$ & Reference & 1.17 & 16.0 & 1.0 \\
\hline WM179 & C. gattii & B & Reference & 4.68 & 16.0 & 0.0625 \\
\hline WM161 & C. gattii & B & Reference & 37.5 & 16.0 & 1.0 \\
\hline
\end{tabular}

FNS, farnesol ( $\mu \mathrm{M})$; FLC, fluconazole $(\mu \mathrm{g} / \mathrm{mL})$; AMB, amphotericin B $(\mu \mathrm{g} / \mathrm{mL}) ;(-)$, not tested.

2004), the farnesol MICs for $C$. neoformans serotypes A, D and $\mathrm{AD}(\mathrm{GM}=6.29 \mu \mathrm{M})$, were higher than those for $C$. gattii serotypes $B$ and $C(G M=4.67 \mu \mathrm{M})$. Additionally, the veterinary strains of $C$. neoformans and $C$. gattii presented higher MIC values ( $\mathrm{GM}=10.91$ and $5.30 \mu \mathrm{M}$, respectively) compared to those of human $C$. neoformans $(\mathrm{GM}=5.56 \mu \mathrm{M})$ and $C$. neoformans and $C$. gattii reference strains $(\mathrm{GM}=2.33$ and $3.30 \mu \mathrm{M}$, respectively). In the present study, antifungal susceptibility profiles of $C$. parapsilosis ATCC 22019 (CLSI, 2008) and Cryptococcus spp. control strains were similar to previously described results (Chong et al., 2010; Hagen et al., 2010; Iqbal et al., 2010; Trilles et al., 2011), hence, validating the accuracy of the methodology employed for susceptibility assays.

In recent years, several studies have shown the antifungal potential of farnesol in vitro against many pathogens (Derengowski et al., 2009; Liu et al., 2010; Weber et al., 2010; Pammi et al., 2011; Brilhante et al., 2012). Nevertheless, the in vivo effect of this compound and therefore its potential as a new antifungal drug - is still a matter of debate. As illustration, one study has shown that $C$. albicans cells pretreated with fluconazole were able

Table 2

Phospholipase activity of Cryptococcus strains following exposure to farnesol.

\begin{tabular}{|c|c|c|c|c|c|c|c|c|}
\hline \multirow{2}{*}{$\begin{array}{l}\text { Farnesol } \\
\text { concentration }\end{array}$} & \multicolumn{4}{|c|}{ C. neoformans } & \multicolumn{4}{|l|}{ C. gattii } \\
\hline & $\begin{array}{l}\mathrm{PZ} \\
\text { Mean } \pm \mathrm{SD}\end{array}$ & $\begin{array}{l}\text { No alteration }{ }^{a} \\
(n)\end{array}$ & $\begin{array}{l}\text { Decreased } \\
\text { activity }^{\mathrm{b}}(n)\end{array}$ & $\begin{array}{l}\text { Increased } \\
\text { activity }^{\mathrm{c}}(n)\end{array}$ & $\begin{array}{l}\mathrm{PZ} \\
\text { Mean } \pm \mathrm{SD}\end{array}$ & $\begin{array}{l}\text { No alteration }{ }^{\mathrm{a}} \\
(n)\end{array}$ & $\begin{array}{l}\text { Decreased } \\
\text { activity }^{\mathrm{b}}(n)\end{array}$ & $\begin{array}{l}\text { Increased } \\
\text { activity }^{\mathrm{c}}(n)\end{array}$ \\
\hline $\mathrm{MIC} / 4$ & $0.94 \pm 0.13$ & 6 & 12 & 3 & $0.75 \pm 0.23$ & 2 & 5 & 8 \\
\hline $\mathrm{MIC} / 2$ & $0.89 \pm 0.20$ & 5 & 9 & 7 & $0.92 \pm 0.14$ & 6 & 5 & 4 \\
\hline MIC & $0.85 \pm 0.22$ & 4 & 9 & 8 & $0.90 \pm 0.19$ & 7 & 6 & 2 \\
\hline
\end{tabular}

PZ, phospholipase activity; SD, standard deviation.

a No variation in enzymatic activity throughout the experiment, regardless the intensity of the response.

b Enzymatic activity variation from positive or strongly positive to negative.

c Enzymatic activity variation from negative to positive or strongly positive. 
to secrete higher concentrations of farnesol and were more lethal in a mouse model (Navarathna et al., 2005). However, according to Hisajima et al. (2008), farnesol protects against candidiasis in mice. Although we could not predict the in vivo effect of farnesol during cryptococcosis, the present study expands the list of fungal pathogens that are inhibited in vitro by this compound. The great susceptibility of Cryptococcus to farnesol points to the antifungal potential of this compound in vivo.

It has been shown that phospholipase and protease production by Cryptococcus spp. is a strain-dependent phenomenon, presenting great frequency and intensity variation (Ma and May, 2009). In the present study, incubation with farnesol was able to change the enzymatic secretion in a strain-dependent manner: some isolates were not affected by the compound, whereas others showed an increase or decrease in enzymatic activities. Statistical differences among groups were not detected and the net effect of farnesol on the test strains was insignificant.

\section{Conclusion}

This study showed, for the first time, that farnesol has an inhibitory effect on strains of the $C$. neoformans species complex, with no relevant change in the secretion of virulence-related exoenzymes. These findings create the perspective for further investigation of the mechanisms through which this molecule inhibits Cryptococcus spp. and the potential use of this compound as an alternative for the treatment of cryptococcosis in the future.

\section{Acknowledgements}

This work was supported by grants from the National Council for Scientific and Technological Development (CNPq; Brazil; Processes 302574/2009-3 and 562296/ 2010-7). We would like to thank the Mycology Laboratory of the Evandro Chagas Clinical Research Institute in Rio de Janeiro (IPEC/FIOCRUZ) for providing the reference strains from its cryptococcal culture collection.

\section{References}

Bien, C.M., Chang, Y.C., Nes, W.D., Kwon-Chung, K.J., Espenshade, P.J., 2009. Cryptococcus neoformans site-2 protease is required for virulence and survival in the presence of azole drugs. Mol. Microbiol. 74, 672-690.

Bovers, M., Hagen, F., Boekhout, T., 2008. Diversity of the Cryptococcus neoformans-Cryptococcus gattii species complex. Rev. Iberoamer. Micol. 25, 4-12.

Brilhante, R.S.N., Paiva, M.A.N., Sampaio, C.M.S., Teixeira, C.E.C., CasteloBranco, D.S.C.M., Leite, J.J.G., Moreira, C.A., Silva, L.P., Cordeiro, R.A., Monteiro, A.J., Sidrim, J.J.C., Rocha, M.F.G., 2011. Yeasts from Macrobrachium amazonicum: a focus on antifungal susceptibility and virulence factors of Candida spp. FEMS Microbiol. Ecol. 76, 268-277.

Brilhante, R.S.N., Valente, L.G.A., Rocha, M.F.G., Bandeira, T.J.P.G., Cordeiro, R.A., Lima, R.A.C., Leite, J.J.G., Ribeiro, J.F., Pereira, J.F., Castelo-Branco, D.S.C.M., Sidrim, J.J.C., 2012. Sesquiterpene farnesol contributing to increased susceptibility to $\beta$-lactams in strains of Burkholderia pseudomallei. Antimicrob. Agents Chemother., http://dx.doi.org/10.1128/ AAC.05885-11.

Cenci, E., Francisci, D., Belfiori, B., Pierucci, S., Baldelli, F., Bistoni, F., Vecchiarelli, A., 2008. Tipranavir exhibits different effects on opportunistic pathogenic fungi. J. Infect. 56, 58-64.
Chayakulkeeree, M., Johnston, S.A., Oei, J.B., Lev, S., Williamson, P.R., Wilson, C.F., Zuo, X., Leal, A.L., Vainstein, M.H., Meyer, W., Sorrell, T.C., May, R.C., Djordjevic, J.T., 2011. SEC14 is a specific requirement for secretion of phospholipase B1 and pathogenicity of Cryptococcus neoformans. Mol. Microbiol. 80, 1088-1101.

Chen, L., Blank, E.S., Casadevall, A., 1996. Extracellular proteinase activity of Cryptococcus neoformans. Clin. Diagn. Lab. Immunol. 3, 570-574.

Chong, H.S., Dagg, R., Malik, R., Chen, S., Carter, D., 2010. In vitro susceptibility of the yeast pathogen Cryptococcus to fluconazole and other azoles varies with molecular genotype. J. Clin. Microbiol. 48, 41154120.

CLSI, 2008. Reference Method for Broth Dilution Antifungal Susceptibility Testing of Yeasts, 3rd ed. Clinical and Laboratory Standards Institute, Wayne, PA (M27-A3).

Cordeiro, R.A., Costa, A.K.F., Brilhante, R.S.N., Lima, R.A.C., Castelo-Branco, D.S.C.M., Ribeiro, J.F., Monteiro, A.J., Rocha, F.A.C., Sidrim, J.J.C., Rocha, M.F.G., 2011. PCR-REA as an important tool for the identification of Cryptococcus neoformans and Cryptococcus gattii from human and veterinary sources. Vet. Microbiol. 154, 180-184.

Costa, A.K.F., Sidrim, J.J.C., Cordeiro, R.A., Brilhante, R.S.N., Monteiro, A.J., Rocha, M.F.G., 2010. Urban pigeons (Columba livia) as a potential source of pathogenic yeast: a focus on antifungal susceptibility of Cryptococcus strains in Northeast Brazil. Mycopathologia 169, 207213.

Derengowski, L.S., De-Souza-Silva, C., Braz, S.V., Mello-De-Sousa, T.M., Báo, S.N., Kyaw, C.M., Silva-Pereira, I., 2009. Antimicrobial effect of farnesol, a Candida albicans quorum sensing molecule, on Paracoccidioides brasiliensis growth and morphogenesis. Ann. Clin. Microbiol. Antimicrob. 8, 13

Hagen, F., Illnait-Zaragozi, M.T., Bartlett, K.H., Swinne, D., Geertsen, E., Klaassen, C.H., Boekhout, T., Meis, J.F., 2010. In vitro antifungal susceptibilities and amplified fragment length polymorphism genotyping of a worldwide collection of 350 clinical, veterinary, and environmental Cryptococcus gattii isolates. Antimicrob. Agents Chemother. 54, 5139-5145.

Hisajima, T., Maruyama, N., Tanabe, Y., Ishibashi, H., Yamada, T., Makimura, K., Nishiyama, Y., Funakoshi, K., Oshima, H., Abe, S., 2008. Protective effects of farnesol against oral candidiasis in mice. Microbiol. Immunol. 52, 327-333.

Hornby, J.M., Jensen, E.C., Lisec, A.D., Tasto, J.J., Jahnke, B., Shoemaker, R., Dussault, P., Nickerson, K.W., 2001. Quorum sensing in the dimorphic fungus Candida albicans is mediated by farnesol. Appl. Environ. Microbiol. 67, 2982-2992.

Hornby, J.M., Nickeerson, K.W., 2004. Enhanced production of farnesol by Candida albicans treated with four azoles. Antimicrob. Agents Chemother. 48, 2305-2307.

Iqbal, N., DeBess, E.E., Wohrle, R., Sun, B., Nett, R.J., Ahlquist, A.M., Chiller, T., Lockhart, S.R., Cryptococcus gattii Public Health Working Group, 2010. Correlation of genotype and in vitro susceptibilities of Cryptococcus gattii strains from the Pacific Northwest of the United States. J. Clin. Microbiol. 48, 539-544.

Jabra-Rizk, M.A., Shirtliff, M., James, C., Meiller, T., 2006. Effect of farnesol on Candida dubliniensis biofilm formation and fluconazole resistance. FEMS Yeast Res. 6, 1063-1073.

Jin, J., Zhang, J.Y., Guo, N., Sheng, H., Li, L., Liang, J.C., Wang, X.L., Li, Y., Liu, M.Y., Wu, X.P., Yu, L., 2010. Farnesol, a potential efflux pump inhibitor in Mycobacterium smegmatis. Molecules 15, 7750-7762.

Kronstad, J.W., Attarian, R., Cadieux, B., Choi, J., D’Souza, C.A., Griffiths, E.J., Geddes, J.M.H., Hu, G., Jung, W.H., Kretschmer, M., Saikia, S., Wang, J., 2011. Expanding fungal pathogenesis: Cryptococcus breaks out of the opportunistic box. Nat. Rev. Microbiol. 9, 193-203.

Kwon-Chung, K.J., Varma, A., 2006. Do major species concepts support one, two or more species within Cryptococcus neoformans? FEMS Yeast Res. 6, 574-587.

Langford, M.L., Hasim, S., Nickerson, K.W., Atkin, A.L., 2010. Activity and toxicity of farnesol towards Candida albicans are dependent on growth conditions. Antimicrob. Agents Chemother. 54, 940-942.

Lester, S.J., Malik, R., Bartlett, K.H., Duncan, C.G., 2011. Cryptococcosis: update and emergence of Cryptococcus gattii. Vet. Clin. Pathol. 40, 4-17.

Lin, X., Heitman, J., 2006. The biology of the Cryptococcus neoformans species complex. Annu. Rev. Microbiol. 8, 69-105.

Liu, P., Luo, L., Guo, J., Liu, H., Wang, B., Deng, B., Long, C.A., Cheng, Y., 2010. Farnesol induces apoptosis and oxidative stress in the fungal pathogen Penicillium expansum. Mycologia 102, 311-318.

Ma, H., May, R.C., 2009. Virulence in Cryptococcus species. Adv. Appl. Microbiol. 67, 131-190.

Meyer, W., Castañeda, A., Jackson, S., Huynh, M., Castañeda, E., IberoAmerican Cryptococcal Study Group, 2003. Molecular typing of IberoAmerican Cryptococcus neoformans isolates. Emerg. Infect. Dis. 9, 189-195. 
Navarathna, D.H.M.L.P., Hornby, J.M., Hoerrmann, N., Parkhurst, A.M., Duhamel, G.E., Nickerson, K.W., 2005. Enhanced pathogenicity of Candida albicans pre-treated with subinhibitory concentrations of fluconazole in a mouse model of disseminated candidiasis. J. Antimicrob. Chemother. 56, 1156-1159.

Pammi, M., Liang, R., Hicks, J.M., Barrish, J., Versalovic, J., 2011. Farnesol decreases biofilms of Staphylococcus epidermidis and exhibits synergy with nafcillin and vancomycin. Pediatr. Res. 70, 578-583.

Price, M.F., Wilkinson, I.D., Gentry, L.O., 1982. Plate method for detection of phospholipase activity of Candida albicans. Sabouraudia 22, 201-207.

Rossignol, T., Logue, M.E., Reynolds, K., Grenon, M., Lowndes, N.F., Butler G., 2007. Transcriptional response of Candida parapsilosis following exposure to farnesol. Antimicrob. Agents Chemother. 51, 2304-2312.

Sharma, M., Prasad, R., 2011. The Quorum-Sensing molecule farnesol is a modulator of drug efflux mediated by $A B C$ multidrug transporters and synergizes with drugs in Candida albicans. Antimicrob. Agents Chemother. 55, 4834-4843.

Semighini, C.P., Murray, N., Harris, S.D., 2008. Inhibition of Fusarium graminearum growth and development by farnesol. FEMS Microbiol. Lett. 279, 259-264.
Shirtliff, M.E., Krom, B.P., Meijering, R.A., Peters, B.M., Zhu, J., Scheper M.A., Harris, M.L., Jabra-Rizk, M.A., 2009. Farnesol-induced apoptosis in Candida albicans. Antimicrob. Agents Chemother. 53, 2392-2401.

Sidrim, J.J.C., Maia, D.C.B.S.C., Brilhante, R.S.N., Soares, G.D.P., Cordeiro, R.A., Monteiro, A.J., Rocha, M.F.G., 2010. Candida species isolated from the gastrointestinal tract of cockatiels (Nymphicus hollandicus): in vitro antifungal susceptibility profile and phospholipase activity. Vet. Microbiol. 145, 324-328.

Trilles, L., Fernandez-Torres, B., Lazera, M.S., Wanke, B., Guarro, J., 2004. In vitro antifungal susceptibility of Cryptococcus gattii. J. Clin. Microbiol. 42, 4815-4817.

Trilles, L., Meyer, W., Wanke, B., Guarro, .J., Lazéra, M., 2011. Correlation of antifungal susceptibility and molecular type within the Cryptococcus neoformans/C. gattii species complex. Med. Mycol. 50, 328-332.

Weber, K., Schulz, B., Ruhnke, M., 2010. The quorum-sensing molecule $E, E$-farnesol - its variable secretion and its impact on the growth and metabolism of Candida species. Yeast 27, 727-739. 